\title{
Procedimento para estruturação da rede de distribuição de ajuda humanitária: o caso da enchente de duque de Caxias-RJ, 2013
}

\author{
Leandro de Oliveira Silva ${ }^{1}$, Renata Albergaria de Mello Bandeira ${ }^{2}$, \\ Vânia Barcellos Gouvêa Campos ${ }^{3}$ \\ 1Programa de Pós-Graduação em Engenharia de Transportes, Ins.tuto Militar de Engenharia, leandro.oliveira@uniube.br \\ 2Programa de Pós-Graduação em Engenharia de Transportes, Ins.tuto Militar de Engenharia, re.albergaria@gmail.com \\ ${ }^{3}$ Programa de Pós-Graduação em Engenharia de Transportes, Ins.tuto Militar de Engenharia, vania@ime.eb.br
}

\section{Recebido:}

25 de setembro de 2017

Aceito para publicação:

15 de maio de 2018

Publicado:

30 de abril de 2019

Editor de área:

Marcio D'Agosto

\section{Palavras-chaves:}

Desastres,

Veículos aéreos não tripulados, Logística humanitária.

\section{Keywords:}

UAV,

Relief distribution,

Capacity planning.

DOI:10.14295/transportes.v27i1.1474

OPEN Access

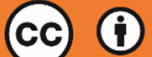

\begin{abstract}
RESUMO
O presente artigo propõe um procedimento de apoio à tomada de decisão em condições operacionais de emergência, na estruturação da rede de distribuição de ajuda, de forma prática e adequada às restrições impostas nos ambientes de pós-desastre. $O$ procedimento é aplicado a uma situação de crise real, tomando como base as características do pós-desastre no município de Duque de Caxias, no estado do Rio de Janeiro, em 2013. Os resultados obtidos envolvem a avaliação da situação da região no pós-desastre, a localização das instalações de atendimento dentro da região afetada, a localização dos centros de abastecimento, o roteamento de veículos e a distribuição das informações em tempo real. Com a aplicação, é possível verificar que o procedimento é capaz de gerar informações em tempo real, bem como auxiliar as equipes de coordenação quanto à escolha dos pontos de atendimento e distribuição dos recursos e organizar as rotas no atendimento às vítimas.
\end{abstract}

\begin{abstract}
This article offers a procedure to support decision-making under emergency operational conditions in the structuring of the aid distribution network in a practical and appropriate way to the restrictions and speed imposed in post-disaster environments. The procedure is applied to a real crisis, taking as a basis the characteristics of the post-disaster in the municipality of Duque de Caxias in the state of Rio de Janeiro in 2013. The results obtained involve the evaluation of the situation of the region in the post-disaster, the location of the service facilities within the affected region, the location of the supply centers, the routing of vehicles and the distribution of information in real time. With the application, it is possible to verify that the procedure is able to generate information in real time, as well as to help the coordination teams in choosing the points of service and distribution of resources and to organize the routes in the care of the victims.
\end{abstract}

\section{INTRODUÇÃO}

Anualmente, cerca de 400 desastres naturais ocorrem ao redor do mundo, afetando aproximadamente 140 milhões de pessoas e gerando inúmeros prejuízos materiais e consequências graves para a população (Guha-Sapir et al., 2015), que está cada vez mais propensa a sofrer a ação de desastres naturais, como furacões, tornados, secas e inundações (Van Wassenhove, 2006). Em 2016, ocorreram 342 desastres naturais no mundo, que causaram cerca de nove mil óbitos, afetaram 564 milhões de pessoas e geraram danos econômicos equivalentes a 154 milhões de dólares (Guha-Sapir et al., 2017). 
Devido a este panorama, as operações humanitárias ganharam destaque na comunidade internacional, assim como as operações logísticas no segmento (Overstreet et al., 2011), por possuírem papel essencial na resposta a desastres (Thomas, 2003). Entretanto, abusos e desperdícios tendem a ocorrer no processo de distribuição até os beneficiários, motivados pela ineficiência e a desigualdade do sistema logístico (McCoy, 2013). Contudo, segundo Thomas e Kopczack (2005) e Van Wassenhove (2006), a eficiência logística pode ser ampliada a partir da otimização do desenho da rede de distribuição, por ser planejada na fase de resposta e agregar a responsabilidade do fluxo de bens e serviços por toda a cadeia.

Ainda, em determinados locais, após um desastre, a acessibilidade de plataformas de manipulação móvel, como veículos terrestres, é impossível (Danko et al., 2014). Assim, um dos principais problemas que ocorrem em uma operação de resposta a desastres é a dificuldade de atualização das informações geográficas locais e localização do ponto geoestratégico (Xu et al.2014). Para sanar esta dificuldade, veículos aéreos não tripulados (VANT) passam a atuar nas operações de resposta a desastres como um recurso alternativo e de apoio à investigação, tal como destacam Xu et al. (2014), OCHA (2014) e Chen et al. (2014). Holguín-Veras et al. (2012) também propõem a adoção de VANT para avaliar o estado das redes de transporte e os locais de entrega, de modo a contribuir para maior eficiência na logística humanitária. Desta forma, realizou-se uma pesquisa bibliográfica sobre as possibilidades de emprego do VANT em operações humanitárias, sendo verificado seu potencial em apoiar a operação de resposta devido à rapidez com que as informações são geradas e disponibilizadas (Xu et al., 2014).

Neste cenário, o presente artigo tem como objetivo propor um procedimento de apoio à decisão para a estruturação da rede de distribuição de ajuda em operações de resposta a desastres de início súbito, com o emprego de VANT e de sistemas de informação geográfica (SIG). Este procedimento envolve a avaliação da situação da região no pós-desastre, a localização das instalações de atendimento dentro da região afetada, a localização dos centros de abastecimento, o roteamento de veículos e a distribuição das informações em tempo real, podendo ser aplicado em condições operacionais de emergência, de forma prática e adequado às restrições e à rapidez impostas pelo ambiente calamitoso.

A partir desta introdução, este trabalho se divide em cinco seções. Na seção 2, apresenta-se a revisão da literatura sobre o emprego do VANT em operações humanitárias. A seção 3 discute o processo de distribuição na logística humanitária. Na seção 4, apresenta-se o procedimento proposto, enquanto a seção 5 analisa os resultados de sua aplicação com base nas características do cenário pós-desastre no município de Duque de Caxias, no estado do Rio de Janeiro, após as enchentes em 2013. Finalmente, na seção 6, encontram-se as conclusões, limitações e sugestões de aprimoramento deste trabalho.

\section{EMPREGO DE VANT NA LOGÍSTICA HUMANITÁRIA}

Imediatamente após um desastre natural, a população afetada precisa ser atendida com suprimentos de sobrevivência e recursos médicos (como água, alimentos, cobertores, abrigo, medicamentos, produtos de higiene e outros). Ainda, a infraestrutura local, que se encontra inutilizada ou bloqueada, deve ser reconstruída para servir de suporte à operação de resposta (Balcik e Beamon, 2008).

Nestas situações, o VANT é uma ferramenta de apoio importante no processo de avaliação e monitoramento da situação no pós-desastre, por permitir a comparação das informações geográficas antes e após a ocorrência de um desastre, tal como destacado nos trabalhos de Krawiec 
et al. (2014), Ocha et al., 2014 e Xu et al. (2014). Contudo, a aplicação do VANT, no contexto humanitário, não se limita a coleta de dados em tempo real, incluindo também o monitoramento da situação atual, atividades de busca e salvamento, detecção de áreas bloqueadas, comunicações, mapeamento, prestação de ajuda e, principalmente, suporte logístico (Meier, 2015; Ocha, 2014; Xu et al., 2014).

Ocha (2014) e Xu et al. (2014) destacam a ação do VANT para identificação de áreas isoladas e de potenciais instalações físicas que possam se tornar pontos de atendimento local como contribuições para a logística humanitária. Exemplos de aplicações de VANT na logística humanitária podem ser percebidos em vários países ao redor do mundo. Murphy et al. (2008) citam a aplicação do VANT no desastre de 2005 no México para diagnosticar o tamanho dos estragos provocados pelo furacão e mensurar as perdas envolvidas. Gong et al. (2010) e Gong et al. (2012) registram a aplicação do VANT, no desastre de 2008 na China, para investigar perigos geológicos e gerar conhecimento para o processamento e construção dos modelos de distribuição de perigo.

Enfim, o emprego das tecnologias de VANT em operação humanitária é estratégico, devido ao uso dos mecanismos de proteção e de manipulação favorecer o atendimento rápido aos beneficiários (Krawiec et al., 2014). Portanto, a melhoria do desempenho dos VANT nas operações humanitárias "tem e deve" ser ampliada. De tal modo, propõe-se, neste trabalho, o uso do VANT para apoio no processo de distribuição na logística humanitária. A seguir, descreve-se como este processo é estruturado.

\section{PROCESSOS DE DISTRIBUIÇÃO NA LOGÍSTICA HUMANITÁRIA}

0 processo logístico de atendimento à população afetada por desastres é iniciado a partir da avaliação da situação local, submetida após o alerta de emergência (socorro) dado pelas autoridades no país afetado ou pela comunidade internacional, dependendo da escala e da gravidade do desastre, no prazo de até 24 horas após a ocorrência do desastre (Anaya-Arenas et al., 2014; Blecken, 2009). A avaliação da situação no pós-desastre é fundamental na transformação das informações em solicitações de recursos necessários à operacionalização da resposta (Balcik et al., 2008), que contará com todas as formas de suprimentos possíveis (Holguín-Veras et al., 2012).

Uma das primeiras decisões a serem tomadas após o reporte da avaliação da situação atual é o projeto da rede de distribuição na área afetada (Holguín-Veras et al., 2012), que consiste na seleção do conjunto de centros logísticos, abrigos e pontos temporários de distribuição local utilizados na ajuda (Anaya-Arenas et al., 2014). A elaboração do projeto da rede de distribuição para resposta é abordada por Balcik et al. (2008), Silva (2011), Holguín-Veras et al. (2012), Costa (2013).

0 projeto da rede de distribuição estabelece um centro de armazenagem primário, preposicionado a um aeroporto ou porto para receber todos os suprimentos de assistência, a partir do qual são destinados para os centros de distribuição regional, onde são armazenados, classificados e transferidos para os pontos de distribuição local (PDD), conforme necessidade (Balcik et al., 2008, Holguín-Veras et al., 2012). A distribuição dos recursos, em volumes fragmentados para as áreas afetadas, é realizada pelos PDDs, que recebem, além dos centros de distribuição (CD) primário, remessas de recursos diretamente de fornecedores locais (Balcik et al., 2008; Silva, 2011). Para a operação humanitária, este ponto de distribuição local é indiferente à esco- 
lha, ou seja, podendo ser uma barraca, uma unidade pré-moldada e até mesmo instalações existentes, como escola, igreja e ginásios (Balcik et al., 2008). Com base nestes conceitos, a seguir é proposto um procedimento para estruturação da rede de distribuição humanitária.

\section{PROCEDIMENTO PARA ESTRUTURAÇÃO DA CADEIA DE DISTRIBUIÇÃO HUMANITÁRIA}

0 procedimento proposto pode ser utilizado como uma ferramenta de apoio para a estruturação da cadeia de distribuição humanitária, na ação de campo. Com a ocorrência do desastre de início súbito, o procedimento subsidiará as equipes de resposta na elaboração da rede de distribuição, seleção das melhores localizações para os pontos de distribuição de ajuda e roteamento de veículos para atendimento à população, a partir de cinco estágios: preparação, mapeamento da região afetada, integração dos dados ao SIG, definição da rede de distribuição e roteirização. A seguir, são detalhadas as atividades relacionadas aos cinco estágios do procedimento.

\subsection{Estágio 1: Preparação}

A preparação é um "estado de prontidão" para responder eventos de crise ou qualquer outro tipo de situação de emergência (Haddow et al., 2011). Seus processos incluem avaliação e verificação da disponibilidade de recursos, bem como a identificação das potenciais fontes de ajuda (Blecken, 2009). 0 Estágio 1 prevê quatro tarefas:

- Identificar e mapear a região em área de risco: A identificação das regiões em áreas de risco (vulnerável) deve seguir as orientações fornecidas pela Defesa Civil (Oliveira, 2016), sendo o mapeamento realizado com o objetivo de posicionar a equipe de coordenação sobre a situação da região, os potenciais locais de atendimento e distribuição, os fatores de vulnerabilidade (TOMASINI \& VAN WASSENHOVE, 2004) e o grau de complexidade para a ação de resposta.

- Avaliar a capacidade de resposta, que trata do planejamento dos meios físicos, institucionais ou sociais (a incluir liderança e gestão) necessários para reduzir os efeitos de um desastre, que deve seguir as diretrizes e o formulário de necessidades propostos pela Defesa Civil (BRASIL, 2013c);

- Criar/atualizar o banco de dados de logística humanitária: são catalogados os recursos disponíveis para a resposta, a incluir equipamentos, locais com potencial de atendimento (como igrejas, escolas, hospitais, áreas de lazer,), reservas ecológicas, agências de proteção (como polícias, bombeiros e defesa civil), centros comunitários e outros. Caso exista um Banco de Dados, tal como proposto por Bastos et al. (2014) e um Sistema de Informações Geográficas (SIG) disponível com informações e recursos necessários para a resposta, a fase de preparação torna o processo de resposta mais ágil e efetivo.

- Organizar e dispor as informações no SIG-T e aguardar o surgimento de uma situação de crise.

Para o caso de haver um desastre em regiões localizadas fora da área de risco, o Estágio 1 não será viável e a elaboração da rede de distribuição terá como base apenas informações da avaliação situacional no pós-desastre, iniciando no Estágio 2.

\subsection{Estágio 2: Mapear região afetada no pós-desastre}

Neste estágio, devem-se considerar três pontos principais: estabelecer os limites de investiga- 
ção da região afetada com base nos condicionantes naturais e antrópicas; escolher tecnologia de investigação; e mapear da região afetada.

0 mapeamento da região permite comparar a situação da região antes e após o desastre com o uso de indicadores para ampliar as estratégias de resposta dentro da região vulnerável (Tomasini \& Van Wassenhove, 2004; Blecken, 2009). Este processo é constituído de três passos: a) plano do voo; b) coleta dos dados; e c) processamento das imagens. Para a estratificação das informações de voo, indica-se o uso do software AgiSoft Photoscan, por possuir um algoritmo computacional de aerotriangulação eficiente e estar orientado para VANT.

\subsection{Estágio 3: Integrar informações ao SIG-T}

Neste estágio, estão previstas as seguintes atividades: (1) acessar o sistema de informações geográficas da região; (2) importar ortofoto, gerada pelo AgiSoft Photoscan; (3) analisar a situação da região antes e após o desastre; (4) identificar áreas afetadas e locais com restrição/obstrução de acesso; e (5) gerar documento de comparação da situação atual.

Após o processo de análise no SIG-T, um documento de comparação da situação atual é criado para atualizar as equipes de campo quanto à situação antes e após a ocorrência do desastre e ampliar as possibilidades de assistência, apontando os acidentes do terreno, edificações importantes, zonas de trabalho, instalações padronizadas, localização dos recursos designados e outras informações necessárias à compreensão da operação.

\subsection{Estágio 4: Definir rede de distribuição de ajuda}

Para o planejamento da rede de distribuição de ajuda humanitária, define-se um referencial espacial com um sistema de coordenadas, baseado em uma malha quadriculada (como a utilizada pelo jogo de "Batalha Naval" e ilustrado na Figura 1a) aplicada sobre o mapa da região atingida, tal como adotado por Jaller e Holguín-Veras (2011), Balcik et al. (2008) e Costa et al. (2016). Assim, a região afetada é dividida em quadrados adjacentes de mesma dimensão, denominados de "Sub-regiões Impactadas" (SRI), cujo dimensionamento considera a distância máxima de caminhada percorrida pelas vítimas como um fator humanitário para reduzir o seu sacrifício em busca de ajuda, considerando as condições do terreno e o esforço para transportar os itens recebidos (Costa et al., 2016). A distância máxima ( $\mathrm{d}_{\max }$ ) de caminhada da população dentro da área afetada corresponde a diagonal deste quadrado (de aresta $\mathrm{l}_{\mathrm{A}} \mathrm{e}$ área $\mathrm{A}_{\mathrm{i}}$ ), definida pela Equação 1. Considera-se, na Equação 1, a velocidade média de caminhada ( $\mathrm{v}_{c}$ ) de uma pessoa em região afetada igual a $4 \mathrm{~km} / \mathrm{h}$, o tempo máximo ( $\mathrm{t}_{\text {máx }}$ ) de 50 minutos e o coeficiente de sinuosidade do trajeto $(\alpha)$ de 1,4 (Costa, 2013). A Figura 1(b) apresenta um exemplo da configuração da SRI.

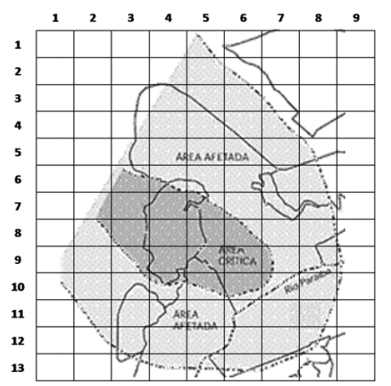

(a)

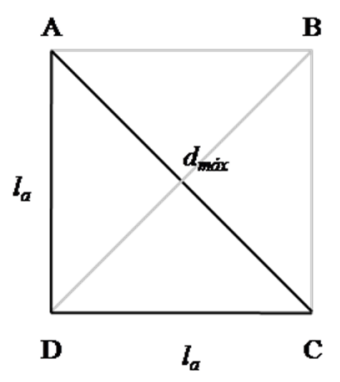

(b)

Figura 1. Região atingida referenciada em áreas impactadas (a) e configuração do quadrante de atendimento humanitário (SRIs) (b). Fonte: Costa (2013) 


$$
d_{\text {máx }}=\frac{v_{c} \cdot t_{\text {máx }}}{\alpha}
$$

O número de beneficiários a serem atendidos em cada SRI é estimado conforme a Equação 2 , considerando-se a densidade demográfica da região $\left(\rho_{\mathrm{r}}\right)$, o tamanho da área mapeada pelo VANT $\left(A_{r}\right)$ e o fator de expectativa de pessoas que recorrerão à ajuda humanitária (definido entre $0,4 \leq \mathrm{f}_{\mathrm{ex}} \leq 0,6$, conforme Ncema, 2009), tal como proposto em Costa et al. (2014).

$$
n_{d}=A_{i} \cdot \rho_{r} \cdot f_{e x}
$$

Considera-se que, em cada SRI, existe apenas um ponto de atendimento aos beneficiários, que caminham até estes pontos para recolher suprimentos. Tal como proposto por Costa et al. (2016), o atendimento pode ser realizado em: (i) pontos de distribuição (PDD): que são instalações fixas selecionadas a partir da infraestrutura existente na SRI (escolas, igrejas, depósitos, etc); ou (ii) pontos de distribuição temporários (CTD): que desempenha a mesma função do PDD, mas em formato temporário com tendas ou veículos grandes, para casos onde não haja infraestrutura disponível em condições de realizar o atendimento adequado. 0 CTD recebe fornecimentos de um PDD de maior capacidade, conforme apresentado na Figura 2, que também mostra o depósito principal de recebimento dos itens de ajuda humanitária, para separação e abastecimento da rede de distribuição, localizado fora da região atingida.

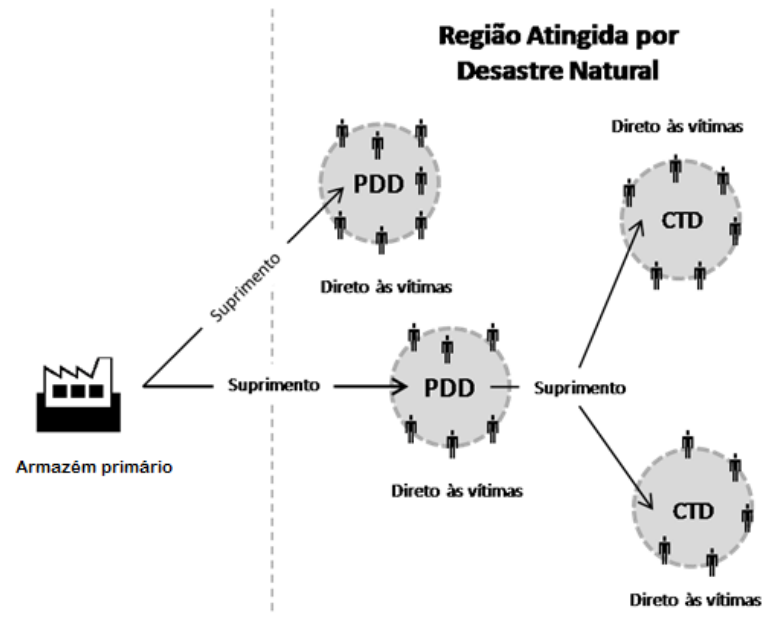

Figura 2. Visão geral do relacionamento entre os elementos da rede de distribuição. Fonte: Costa et al. (2016)

Para projeção da demanda de abastecimento para os beneficiários em cada SRI, adota-se o procedimento proposto por Costa et al. (2014), o qual considera que os itens de ajuda distribuídos (alimento, água, cobertor e itens de higiene) são entregues em embalagens por classe de item em quantidade suficiente para a necessidade diária de uma pessoa. Assim, considerando a cota diária para uma pessoa da classe de ajuda e o número de beneficiários de cada SRI, definese a capacidade mínima necessária de armazenamento para atendimento ( $\mathrm{C}_{\mathrm{a}}$ ) (Equação 3) e a área mínima necessária $\left(\mathrm{A}_{\mathrm{a}}\right)$ para os pontos de distribuição de cada SRI (Equação 4). Tal como em Costa et al. (2014), considera-se o fator de cobertura dos estoques ( $\mathrm{f}_{\mathrm{e}}$ ) de 1,5 dias e o fator de movimentação ( $\mathrm{f}_{\mathrm{m}}$ ) igual a 30\% (SERT, 2010); a cota diária para uma pessoa (consumo/dia) é de 0,540 kg de alimento, 8 unidades de $500 \mathrm{ml}$ de água, 1 unidade de 1,32 kg de cobertor e $0,260 \mathrm{~kg}$ de kit higiene, conforme referência padrão adotada no manual do PAHO (2000).

$$
\begin{gathered}
C_{a}=\left(\sum_{j} n_{d} \cdot c_{j}\right) \cdot f_{e} \\
A_{a}=\left(\sum_{j} \frac{n_{d} \cdot c_{j}}{q_{j}} \cdot e_{j}\right) \cdot f_{e} f_{m}
\end{gathered}
$$


Após a definição da área mínima para cada PDD, identificam-se, na região afetada, instalações que podem atuar como centro de distribuição primário (CD) ou PDD, bem como se verifica a necessidade de CTD para montagem da rede de distribuição. A montagem da rede de distribuição é abordada em três etapas de localização: (i) definição dos PDDs para atendimento aos beneficiários das SRI; e (ii) definição do atendimento aos beneficiários das SRI através de CTDs, quando necessário, designando PDDs como pontos centrais de reabastecimento; e (iii) definição dos CDs para atendimento aos PDDs.

Conforme proposto em Costa et al. (2016), os PDDs selecionados devem ser, de preferência, locais que a população local está familiarizada e, caso haja mais de um potencial PDD em uma SRI, deve-se escolher aquele de maior capacidade de armazenagem. PDDs cuja área puder atender a demanda de, no mínimo, mais uma SRI se candidatam a ser fornecedor de recursos para atender o CTD. Para a alocação dos CTDs a estes PDDs, Costa et al. (2016) propõem duas estratégias: (a) utilizar o menor número de PDDs no abastecimento de todos os CTDs; ou (b) utilizar a menor distância total para o abastecimento dos locais de atendimento à população.

Ao se optar pela estratégia que seleciona o menor número de PDDs, deve-se adotar a técnica de backtracking proposta por Costa (2013) para determinar o conjunto Isel com as combinações de instalações sob a forma dos subconjuntos de I, cuja soma das capacidades $\mathrm{c}_{\mathrm{i}}$ dos elementos de cada subconjunto seja capaz de cobrir a todas as instalações do conjunto J (i.e., $\sum_{\mathrm{i}} \mathrm{c}_{\mathrm{i}} \geq \mathrm{m}$ ), sendo: $\mathrm{I}=\{\mathrm{i} \mid \mathrm{i}=$ PDDs com capacidade disponível para atender Sub-regiões Impactadas; $\mathrm{i}=$ $1 \ldots \mathrm{n}\} ; \mathrm{J}=\{\mathrm{j} \mid \mathrm{j}=$ Sub-regiões Impactadas em que não haja PDD para atendimento; $\mathrm{j}=1 . . . \mathrm{m}\}$; e $\mathrm{I}_{\text {sel }}$ = conjunto dos subconjuntos de I cuja soma das capacidades dos seus elementos é maior ou igual ao total da área necessária para atender às Sub-regiões Impactadas $\mathbf{j} \in \mathrm{J}$. A partir deste resultado, identifica-se o conjunto $I_{\min }$ com os elementos de $I_{\text {sel }}$ de menor dimensão (número de instalações), garantindo o menor número de PDDs para suporte aos CTDs. Caso o conjunto I $\min$ tenha dois ou mais elementos, há mais de uma solução; e para decisão consideram-se as distâncias a serem percorridas por cada elemento (conjunto de PDDs) do conjunto $I_{\min }$ aos respectivos CTDs (Costa, 2013).

Ao se optar pela estratégia que privilegia a menor distância, aplica-se o método de solução do "problema clássico de transportes" para minimização dos custos de distribuição, neste caso considerando os custos como a soma das distâncias entre os PDDs ( $i$ ) e as áreas CTDs ( $j$ ) atendidas $\left(\mathrm{d}_{\mathrm{ij}}\right)$, garantindo o atendimento à todas as SRIs por apenas um PDD (Costa, 2013). Para a modelagem, considera-se a distância euclidiana com a aplicação de um fator de correção equivalente a 1,4, referente à sinuosidade das vias e o tráfego no cálculo da distância percorrida, corrigindo a linha reta (Euclidiana) em distância real, conforme recomendado por Alvarenga e Novaes (2001).

A partir do posicionamento dos PDDs e CTDs, o processo de localização dos CDs é iniciado. A escolha consiste na identificação de locais fora da área das SRIs que tenham capacidade suficiente para abastecer o maior número possível de PDDs, mas dentro do limite de um raio de atuação significativo, que limite a distância máxima às SRIs.

O primeiro passo para a localização dos CDs consiste na construção de $k$ grupos (clusters) de PDDs e CTDs agrupados por similaridade de menor distância, e a determinação de pontos de equilíbrio (centroides). Para tanto, utiliza-se o algoritmo de agrupamento interativo $k$-means (Balcik et al., 2010; Faria et al., 2015). Assim, após a definição dos $k$ centroides dos $k$ grupos, um $z$ centroide é calculado para representar o centroide dos centroides dos agrupamentos. Este 
ponto é a base para a definição de duas faixas de localização (elipse) máxima e mínima de atuação dos potenciais CDs (Faria et al., 2015) à rede de distribuição. A faixa do limite mínimo e o limite máximo devem ser definidas pelo usuário por dependerem do relevo da região e da área que afetada. Estas faixas de atuação têm a função de determinar quais CDs estão localizados próximos às SRIs, mas garantindo que estão dentro de uma área segura. Em seguida, são calculadas as distâncias euclidianas entre os candidatos a CDs e os centroides, sendo que a localização dos CDs será dada pelas menores distâncias (Faria et al., 2015).

\subsection{Estágio 5: Roteirização}

O problema de roteirização analisa a localização dos veículos em determinados depósitos centrais (i), e são programados para visitar os pontos específicos para atender demanda (j), relacionando capacidade, distância, custo e tempo. A roteirização é realizada no SIG-T, por meio do algoritmo usual do método heurístico das economias de Clarke e Wright (com janelas de tempo), considerando que os veículos são carregados com a máxima capacidade possível e as paradas sequenciadas e sem sobreposição.

\section{APLICAÇÃO DO PROCEDIMENTO PARA ESTRUTURAÇÃO DA CADEIA DE DISTRIBUIÇÃO HUMANITÁRIA: O CASO DE DUQUE DE CAXIAS}

Esta seção apresenta a aplicação do procedimento proposto para o evento calamitoso ocorrido no Município de Duque de Caxias no Estado do Rio de Janeiro, Brasil, e reconstrói o cenário de crise para aplicar, de forma ordenada, os cinco estágios do procedimento.

\subsection{Caracterização do evento de crise}

Entre os dias 02 e 03 de janeiro de 2013, o município de Duque de Caxias no Estado do Rio de Janeiro, Brasil, registrou um índice de 212 milímetros de precipitação pluviométrica. A lâmina d'água de 4 a 5 metros de altura transbordou o Rio Saracuruna, provocando eventos de enxurrada no Rio Capivari e nos córregos da região, afetando os Distritos de Xerém, Imbariê e Campos Elíseos, pertencentes ao município de Duque de Caxias (Brasil, 2013). O Distrito de Xerém foi o mais afetado, sendo registradas duas mortes, 60 feridos, 270 desabrigados, 1.126 desalojados e 50.000 afetados no pós-desastre (Brasil, 2013).

A ação de resposta ao evento foi iniciada pela população local, retirando e salvando as famílias próximas às margens do rio Capivari, seguido do apoio da prefeitura de Duque de Caxias, Defesa Civil, militares e instituições religiosas. A Prefeitura estabeleceu um gabinete de crise no Distrito de Xerém 13 horas após o evento, com 100 homens, 20 caminhões e 10 tratores a fim de apoiar a operação de ajuda. A Igreja Wesleyana, localizada a 17 minutos do centro de Xerém, foi definida como o centro de distribuição regional, responsável por receber e distribuir as doações até os pontos de atendimento dentro do distrito mobilizado pela Defesa Civil (SEDEC), Corpo de Bombeiros (CBMERJ), Guarda Municipal, Prefeitura de Duque de Caxias, Bancos e Igrejas.

Os desafios relacionados a esta operação de resposta não foram apenas de ordem material e econômica, mas também imaterial e subjetiva (Souza et al., 2014). Em entrevista com os oficiais da SEDEC e CBMERJ que atuaram diretamente no desastre, os principais problemas enfrentados no processo de atendimento à região foram: a ausência dos planos de prevenção e de preparação que pudessem dar suporte à fase do pós-desastre; lentidão do processo de identificação das 
áreas afetadas, assim como do reconhecimento dos pontos de isolamento; e a ausência de critério para a localização dos centros de distribuição e estimação dos suprimentos de ajuda, que foram insuficientes para atender a população vulnerável.

A partir deste cenário, o procedimento é implementado nas subseções a seguir reconectando a realidade dos envolvidos às necessidades de resposta. Para tanto, o procedimento terá como objetivo solucionar os problemas críticos de coordenação das ações da SEDEC/CBMERJ a partir da sugestão de um plano de trabalho de reconhecimento dos locais afetados de forma integrada, de estimação da demanda e definição da rede de distribuição de ajuda a ser implementada no pós-desastre.

\subsection{Aplicação do procedimento}

\subsubsection{Mapeamento da região afetada no pós-desastre}

Devido à incapacidade em antever o evento de crise, que assolou o município de Duque de Caxias em 2013, o Estágio 1, que tem como finalidade preparar as regiões em situação vulnerável para responder aos eventos de crise, não foi acionado. Assim, os Estágios 2 e 3 assumem a responsabilidade de preparar todas as informações necessárias para colocar o procedimento em ação, conforme apresentado nesta subseção.

0 mapeamento da região no pós-desastre é direcionado à investigação das áreas de risco a partir do centro do Distrito de Xerém. Para o mapeamento, utilizou-se o VANT Horus FT-100, com apoio de um plano de voo, que inclui a escolha o tipo de sensor de câmera, o menor tamanho dos objetos de investigação (GSD $=20 \mathrm{~cm})$, a altura de voo $(598 \mathrm{~m})$, e a restituição dos dados em um mapa georreferenciado para uso no SIG.

A restituição da geometria do Distrito de Xerém foi realizada a partir da construção do modelo poligonal (texturização) por meio do software AgiSoft Photoscan. Em seguida, a ortofoto georreferenciada, resultante da restituição, é importada ao SIG-T, sendo ativada a ferramenta Google Hybrid, de modo a possibilitar a comparação à situação anterior à ocorrência do desastre.

As tarefas de quantificação e cadastramento dos recursos necessários à ajuda devem ser realizadas paralelas às tarefas de identificação e mapeamento, para acelerar a execução do procedimento. Para o caso analisado, foram identificadas 35 instalações candidatas a PDDs e 3 candidatas a CDs, cujas localizações (longitude e latitude), descrições e funções foram cadastradas no arquivo "cadastro de locais de apoio" do Banco de Dados de Logística Humanitária. Também foram registrados (arquivo "cadastro de veículos") 12 veículos, disponíveis na região afetada, com as informações de capacidade, disponibilidade, tipo e custo operacional. Dos 12 veículos disponíveis, um é do tipo 2 eixos, articulado, com 16,5 m de comprimento e capacidade bruta de $26 \mathrm{t}$; três são do tipo 3 eixos, rígido, com $12 \mathrm{~m}$ de comprimento e capacidade bruta de $18 \mathrm{t}$; quatro são do tipo 2 eixos, 6 rodas $(6 \times 2)$ e capacidade bruta de 7,5 t; e quatro são do tipo 2 eixos, 4 rodas $(4 \times 2)$ e capacidade de $3,5 \mathrm{t}$.

\subsubsection{Definição da rede de distribuição de ajuda}

Para construção da rede de distribuição, inicialmente, calculou-se a distância máxima de caminhada para dimensionamento das sub-regiões impactadas (SRIs) e verificou-se a densidade demográfica média da região impactada com as informações disponíveis do último censo do IBGE (2015b). A distância máxima de caminhada é calculada pela Equação 1, considerando um coeficiente $(\alpha)$ igual a 1,4 e que uma pessoa em área afetada caminha a uma velocidade média $\left(\mathrm{V}_{c}\right)$ 
de $4 \mathrm{~km} / \mathrm{h}$ a um tempo máximo ( $\mathrm{t}_{\text {máx }}$ ) de 50 minutos. Logo, a diagonal do quadrado correspondente a SRI deve ter $2,38 \mathrm{~km}[=(4 \times 50 \div 60) \div 1,40]$, seus lados têm dimensão igual a 1,68 km $[=(2,38 \div \sqrt{2})]$ e a área de cada SRI equivale a $2,8344 \mathrm{~km}^{2}\left[=(2,38 \div \sqrt{2})^{2}\right]$. Assim, aplica-se o referencial espacial sobre o mapa da região, criando $16[=(46,24 \div 2,8344]$ SRIs para a área da unidade territorial mapeada igual a $46,24 \mathrm{~km}^{2}[=6,8 \times 6,8]$, tal como ilustrado na Figura 3 .

Com base na densidade geográfica $\left(\rho_{\mathrm{r}}=1.322 \mathrm{hab} . / \mathrm{km}^{2}\right)$ e adotando-se o fator de expectativa de pessoas que recorrerão à ajuda $\left(\mathrm{f}_{\mathrm{ex}}\right)$ de $60 \%$, estima-se o número de habitantes de cada SRI que requisitará ajuda nos locais de atendimento ( $n_{d}$ ) em 2.247 habitantes (Equação 2). Logo, a quantidade e a necessidade de armazenamento para atendimento são calculadas segundo a cota diária para uma pessoa da classe de ajuda indicada pelo PAHO (2000). Os resultados deste processo são apresentados na Tabela 1.

Tabela 1. Capacidade de armazenamento para atendimento $\left(\mathrm{m}^{2}\right)$, prevendo 2.247 habitantes.

\begin{tabular}{llllll}
\hline Classe de Ajuda & Alimento & Água & Cobertor & Higiene & TOTAL \\
\hline Cota/dia $(\mathrm{kg})$ & 0,54 & 4,20 & 1,32 & 0,26 & \\
Área por unidade de armazenagem $\left(\mathrm{m}^{2}\right)$ & 1,32 & 0,96 & 1,20 & 0,93 \\
Peso por unidade de armazenagem $(\mathrm{kg})$ & 480,00 & $\mathbf{7 8 5 , 0 0}$ & $1.188,00$ & 540,00 & \\
Necessidade/dia (kg) & $\mathbf{1 . 2 1 3 , 3 8}$ & $\mathbf{9 . 4 7 3 , 4 0}$ & $\mathbf{2 . 9 6 6 , 0 4}$ & $\mathbf{5 8 4 , 2 2}$ & $\mathbf{1 4 . 2 0 1 , 0 4}$ \\
Fator de cobertura (dias) & 1,50 & 1,50 & 1,50 & 1,50 & \\
Quantidade necessária para armazenamento $(\mathrm{kg})$ & $1.820,07$ & $14.156,10$ & $4.449,06$ & 876,33 & $21.301,56$ \\
Necessidade/unidade armazenada & 4 & 19 & 4 & 2 & \\
Cobertura de estoque (dias) & 1,58 & 1,58 & 1,60 & 1,85 & \\
Fator de movimentação & $30 \%$ & $30 \%$ & $30 \%$ & $30 \%$ & \\
Área necessária armazenamento diário $\left(\mathrm{m}^{2}\right)$ & 7,54 & 26,06 & 6,86 & 2,66 & 43,11 \\
\hline
\end{tabular}

Em paralelo, o banco de dados com o cadastro das instalações candidatas a CD ou PDD são importadas para o SIG-T como uma base de pontos (layer pontos) com a descrição dos locais, bem como os dados de acessos rodoviários à região afetada, destacando ruas, avenidas, estradas e rodovias como base de linhas (layer linhas). Em seguida, a ortofoto (mapa da situação atual) foi sincronizada às sobreposições do SIG-T no intuito de avaliar quais acessos aos possíveis locais de atendimento à população estavam bloqueados ou inviabilizados após o desastre. Os locais "inviabilizados" foram retirados do planejamento e atualizados na Base de Dados de Logística Humanitária, de modo que apenas 12 instalações foram identificadas como candidatas a pontos de distribuição (potenciais PDDs), e 3 locais como candidatos a centro de distribuição (potenciais CDs), como apresentado na Tabela 2. A decisão de classificar uma instalação como potencial PDD ou CD se baseou no tamanho do espaço físico disponível no local e em sua capacidade de atendimento, conforme apresentado na seção 3.4

As instalações candidatas a CDs e PDDs (tabela 2) são localizadas de acordo com suas coordenadas, enquanto os CTDs são localizados no ponto central de cada SRI, com as suas localizações definidas pela linha e a coluna do referencial espacial, como apresentado na Figura 3. A Tabela 3 apresenta cada SRI, identificando se esta possui ou não infraestrutura, classificando PDD para SRIs com infraestrutura e CTD para SRIs sem infraestrutura.

Em seguida, para SRIs com infraestrutura identificou-se como um PDD as instalações com área disponível maior ou igual ao espaço necessário para guardar a quantidade necessária ao atendimento; PDDs com sobra de capacidade se candidatam a um possível fornecedor de recursos para atender CTD; e, caso haja mais de uma instalação na SRI, é escolhida apenas a de maior capacidade de armazenagem. 
Tabela 2. Relação dos locais disponíveis para aturar na resposta como PDD

\begin{tabular}{llllll}
\hline Identificador & Instalação - ID & Longitude & Latitude & Descrição Local & Situação \\
\hline & 1 & -43308223 & -22572284 & Campo de Esporte & Disponível \\
& 2 & -43302006 & -22579437 & Atacadista & Disponível \\
& 3 & -43303586 & -22579087 & Campo aberto & Disponível \\
Potencial PDD & 4 & -43304439 & -22592565 & Campo Aberto & Disponível \\
& 6 & -43307269 & -22595965 & Campo Aberto & Disponível \\
& 7 & -43294834 & -22598629 & Campo Aberto & Disponível \\
& 8 & -43295789 & -22599253 & Campo de Esporte & Disponível \\
& 9 & -43283644 & -22589120 & INMETRO & Disponível \\
& 10 & -43262079 & -22585940 & Laticínio & Disponível \\
& 11 & -43286728 & -22568425 & Campo Aberto & Disponível \\
& 12 & -43300083 & -22580821 & Campo Aberto & Disponível \\
& 1 & -43295440 & -22600714 & Campo Aberto & Disponível \\
& 2 & -43304302 & -22581641 & Escola & Disponível \\
& 3 & -4328364 & -2258912 & INMETRO & Disponível \\
& 12 & -4318209 & -2235154 & Igreja & Disponível \\
\hline
\end{tabular}

Tabela 3. Identificação de PDD ou CTD nas SRIs

\begin{tabular}{llllll}
\hline Localização SRIs & Infraestrutura & Identificação & Localização SRIs & Infraestrutura & Identificação \\
\hline$(1,1)$ & NÃO & CTD & $(3,1)$ & NÃO & CTD \\
$(1,2)$ & SIM & PDD & $(3,2)$ & SIM & PDD \\
$(1,3)$ & SIM & PDD & $(3,3)$ & SIM & PDD \\
$(1,4)$ & NÃO & CTD & $(3,4)$ & NÃO & área de mata \\
$(2,1)$ & SIM & PDD & $(4,1)$ & NÃO & CTD \\
$(2,2)$ & SIM & PDD & $(4,2)$ & SIM & PDD \\
$(2,3)$ & SIM & PDD & $(4,3)$ & NÃO & área de mata \\
$(2,4)$ & NÃO & CTD & $(4,4)$ & NÃO & CTD \\
\hline
\end{tabular}

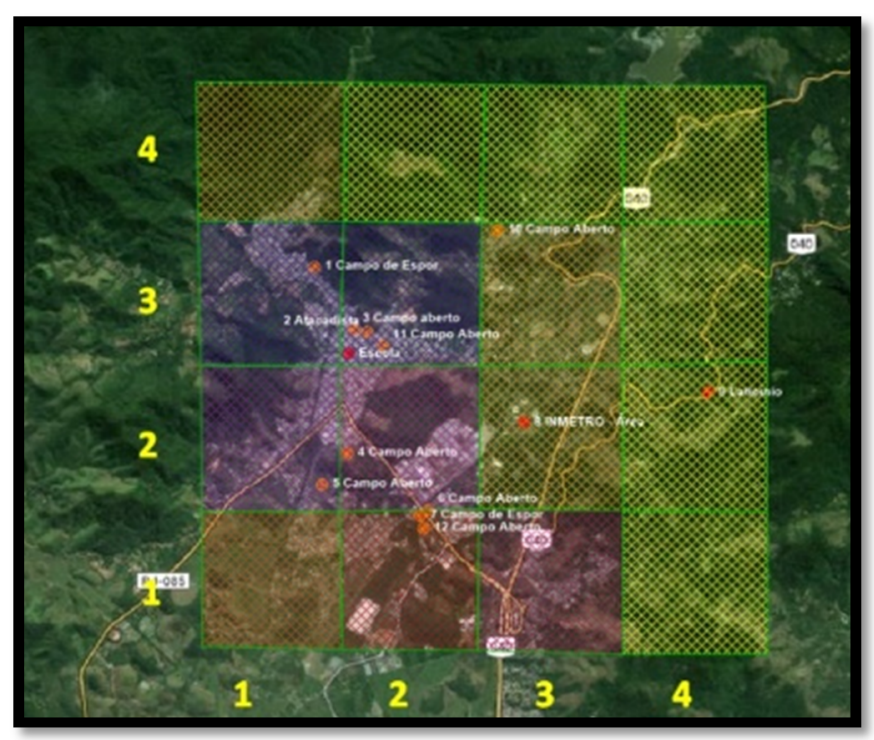

Figura 3. Identificação dos PDD e CTD nas SRIs

A rede de distribuição entre PDDs e CTDs é criada considerando que a distância entre os pontos é euclidiana com a aplicação de um fator de correção $(\alpha)$ igual a 1,4. A solução da rede 
de distribuição privilegia o menor número de CDs e a menor distância total para o abastecimento dos locais de atendimento à população. As restrições impostas para a solução consideram as limitações impostas no procedimento, citadas na seção 3.4, onde CTDs são abastecidos apenas por PDD. 0 resultado final da rede é destacado na figura 4.

A partir da definição dos PDDs e CTDs, o processo de escolha dos CDs é iniciado utilizando o algoritmo de agrupamento interativo $k$-means, sobre os potenciais CDs listados na Tabela 2, com a definição das faixas de localização (elipses) mínima do raio de atuação dos CDs igual a 6,8km, de modo a garantir que os CDs estejam fora das SRIs. A faixa de localização máxima foi definida como a maior distância do z centroide ao potencial CD. Este processo é ilustrado na Figura 4. Ao final, apenas um CD é apresentado como viável ao processo de atendimento às SRIs, localizado

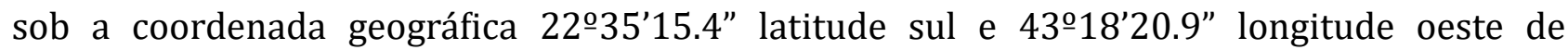
Greenwich. Destaca-se que este foi o mesmo depósito selecionado empiricamente no caso real.

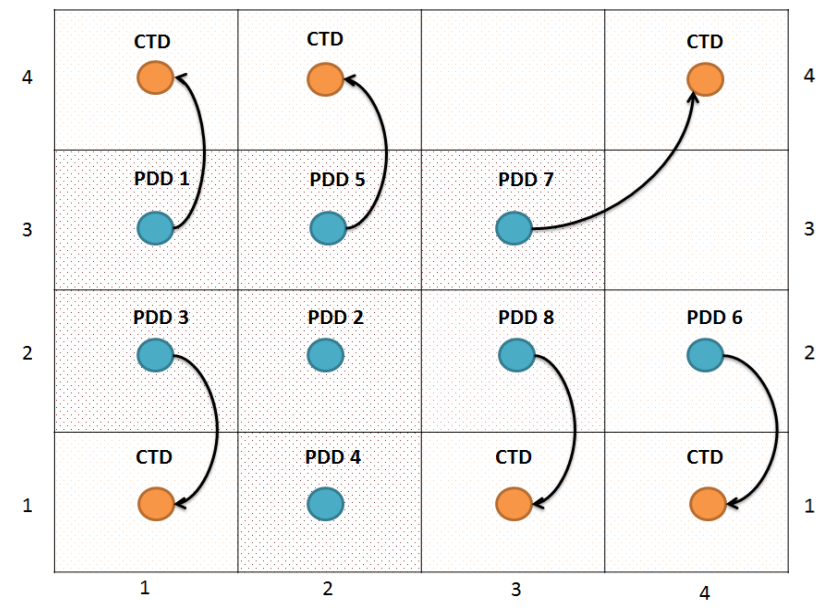

(a)

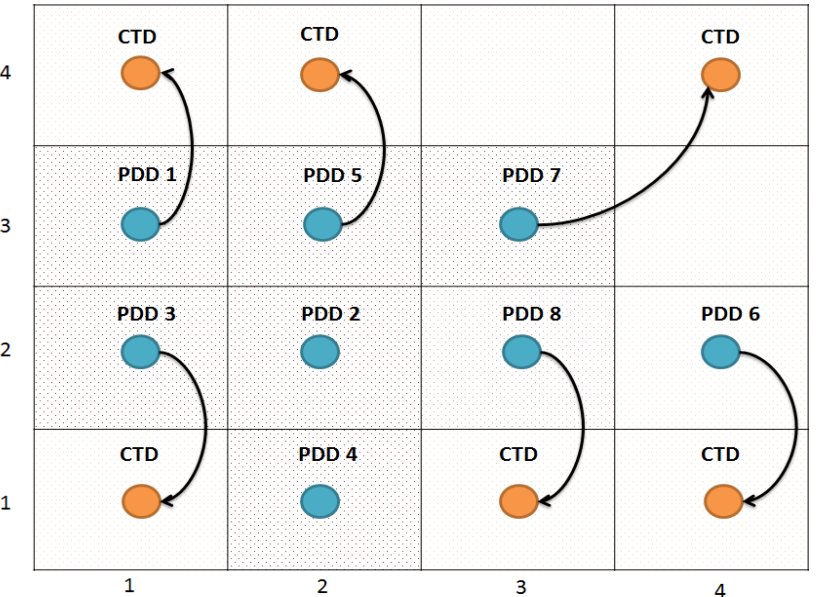

(b)

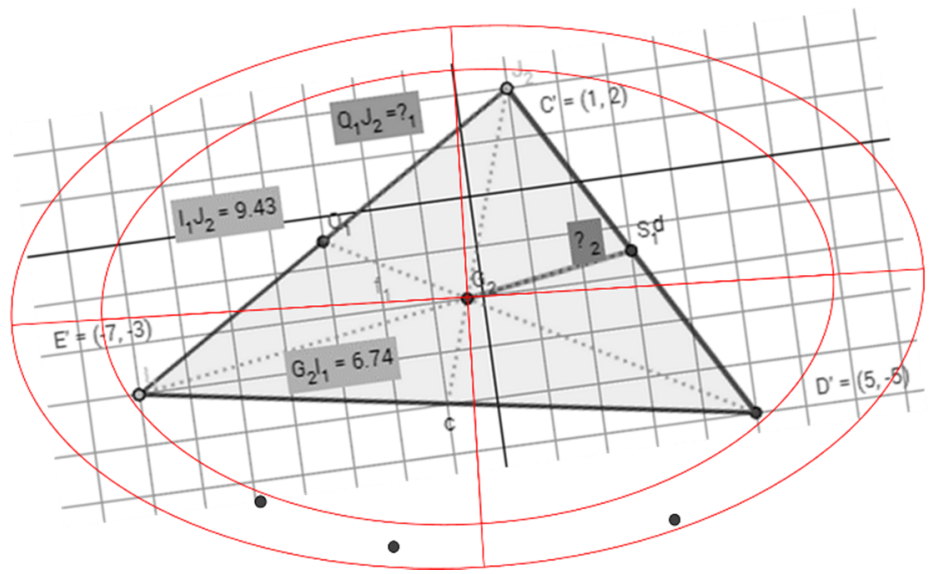

(c)

Figura 4. Ilustração do resultado da rede de distribuição (a), localização dos $k$ centroides entre PDDs e CTDs (b) e localização do $z$ centroide e determinação das faixas máximas e mínimas de atuação significativo dos CD (c).

\subsubsection{Roteirização}

A roteirização dos veículos na rede de distribuição é o último estágio do procedimento, com a missão de selecionar as melhores rotas na rede e minimizar os custos de distribuição entre CDs, PDDs e CTDs por meio do SIG-T. 
Para iniciar o processo de roteirização, os arquivos "locais de apoio" e "cadastro de veículos" (12 veículos disponíveis inicialmente) do Banco Dados de Logística Humanitária são importados ao SIG-T, contendo, além da localização dos PDD e CTD, as informações de demanda local, janela de entrega e tempo de atendimento. Em seguida, cria-se a matriz de roteamento (origem / destino), com o objetivo de estabelecer os parâmetros entre a distância e o tempo de viagem entre o CDs, PDDs e CTDs. Finalmente, utilizando a rotina Vehicle Routing do SIG-T, o problema de roteamento é solucionado, tendo como resultado caminhos convertidos em um arquivo geográfico de rotas sobre um mapa. Destaca-se que apenas 8 dos 12 veículos disponíveis são suficientes para a operação de abastecimento, ou seja, para o trabalho de abastecimento são selecionados 4 veículos do tipo 2 eixos, 6 rodas (6x2) e capacidade bruta de 7,5 toneladas e 4 veículos do tipo 2 eixos, 4 rodas ( $4 \times 2$ ) e capacidade bruta de 3,5 toneladas.

\section{CONSIDERAÇÕES FINAIS}

O procedimento proposto neste artigo visa auxiliar os tomadores de decisão a estruturar a rede de distribuição em operações de resposta a desastres. Cada estágio do procedimento foca processos decisórios de projeto de rede, de maneira intrínseca, partindo da definição de uma estratégia de cadeia (Estágio 2) da configuração das instalações distribuidoras (centros de distribuição) e locais desejáveis de atendimento (Estágio 3), escolhas de localização e alocação de capacidade (Estágio 4) e roteamento de abastecimento (Estágio 4).

Em observação à aplicação do procedimento, refazendo o projeto de rede de distribuição do desastre ocorrido em Duque de Caxias, é possível constatar que o procedimento atende às especificações no tempo de avaliação da situação no pós-desastre descrito por Anaya-Arenas et al. (2014), Banomyong et al. (2009) e Blecken (2009). Os autores citam que a avaliação da situação da região afetada (pós-desastre) deva ser concluída no prazo de até 24 horas após a ocorrência do desastre. Assim, fica estabelecido que o procedimento deve ser aplicado imediatamente após a ocorrência do desastre, a contar do alerta de emergência (socorro) dado pelas autoridades no país afetado ou pela comunidade internacional, dependendo da escala e da gravidade do desastre/crise.

Outro ponto a ser destacado é a redução do tempo de decisão e preparação das ações de resposta, oportunizadas pela integração VANT-SIG-T e as estimativas de demanda. Na situação real, os recursos de suprimentos e os locais de atendimento à população (PDDs, CTDs e CD) foram oficialmente determinados num intervalo superior a 36 horas (após a ocorrência do desastre); e, em comparação ao procedimento, os recursos de suprimentos, PDDs, CTDs e CD foram estimados e localizados em um período de 17 horas (considerando o tempo de preparação e voo do VANT, tratamento das imagens e aplicação de todas as etapas do procedimento no SIG). Além disto, o processo permitiu a redução do número de veículos utilizados para a realização da distribuição (no caso real, foram utilizados 12 veículos, sendo que, com a aplicação do procedimento, apenas oito destes veículos seriam suficientes para atender a demanda). Com relação ao depósito central, destaca-se que o ponto escolhido foi o mesmo do caso real.

Enfim, após a finalização do procedimento para localização de centros de distribuição e roteamento de veículos é possível observar uma ação de estruturação dos locais de atendimento em menor tempo maximizado pelo atendimento ao maior número de indivíduos à prestação de ajuda. Dos resultados encontrados, é destacado o auxílio às atividades de armazenagem (posicionamento e destinação), alocação (relacionamento da demanda das regiões afetadas aos em- 
barques) e entrega (seleção de veículo-rota relacionado com as quantidades - produtos enviados a cada localização), respeitando a capacidade instalada em cada PDD e em cada veículo utilizado para efetuar o abastecimento diário à população.

\section{AGRADECIMENTOS}

O presente trabalho foi realizado com apoio da Coordenação de Aperfeiçoamento de Pessoal de Nível Superior - Brasil (CAPES) - Código de Financiamento 001.

\section{REFERÊNCIAS}

Anaya-Arenas, A. M.; Renaud, J.; Ruiz, A. (2014). Relief distribution networks: a systematic review. Annals of Operations Research, v. 223, p. 53-79. DOI: 10.1007/s10479-014-1581-y

Balcik, B.; Beamon, B. M.; Smilowitz, K. (2008). Last mile distribution in humanitarian relief. Journal of Intelligent Transportation Systems, V. 12, p. 51-63. DOI: .1080/15472450802023329

Balcik, B.; Beamon, B. M. (2008). Facility location in humanitarian relief. International Journal of Logistics Research and Applications, v. 11, p.101-21. DOI: 10.1080/13675560701561789

Balcik, B.; B. M. Beamon; C.C. Krekci.; Muramatsu, M.; Ramirez, M. (2010). Coordination in humanitarian relief chains: Practices, challenges and opportunities. International Journal Production Economics. Science Direct, v. 126, p. 22-34. DOI: 10.1016/j.ijpe.2009.09.008

Banomyong, R.; Bersford, A.; Pettit, S. (2009). Logistics relief response model: the case of Thailand's tsunami affected area. International Journal of Services Technology and Management, v.12, n.4, p. 414. DOI: 10.1504/IJSTM.2009.025816

Bastos, M. A. G. (2013). Procedimento logístico para operação de resposta a desastres. Dissertação de mestrado. Instituto Militar de Engenharia, Rio de Janeiro.

Brasil. Ministério da Integração Nacional. Política Nacional de Defesa Civil. Brasília, DF: Secretaria Nacional de Defesa Civil, 2007.

Chen, Y; Zhang, H; Xu, M. (2014). The coverage problem in UAV network: a survey. Proceedings of the International Conference on Computing, Communication and Networking Technologies (ICCCNT '14), p. 1-5, IEEE, Hefei, China. DOI: 10.1109/ICCCNT.2014.6963085

Costa, S. R. A. (2013). Procedimento para estruturação do processo de distribuição em operações de logística humanitária. Dissertação de Mestrado. Instituto Militar de Engenharia, Rio de Janeiro.

Costa, S.R.A, Bandeira, R.A.M, Campos, V.B.G. (2016). Um procedimento para a estruturação da rede de distribuição de ajuda para ações humanitárias em desastres naturais. Anais do XXX Congresso de Pesquisa e Ensino em Transportes, ANPET, Rio de Janeiro.

Costa, S.R.A, Bandeira, R.A.M, Campos, V.B.G. (2014). Pontos de distribuição na logística humanitária: demanda de produtos e aspectos operacionais. Anais do XXVIII Congresso de Pesquisa e Ensino em Transportes, ANPET, Curitiba.

Danko, Todd W.; Oh, Paul Y. (2014). Design and Control of a Hyper-Redundant Manipulator for Mobile Manipulating Unmanned Aerial Vehicles. Journal of Intelligent \& Robotic Systems, v. 73, n. 1-4, p. 709-723. DOI: 10.1007/s10846-013-9935-2

Guha-Sapir, D. V.; Below, F. R.; Ponserre, S. (2015). Annual Disaster Statistical Review 2014. Centre for Research on the Epidemiology of Disasters (CRED). Institute of Health and Society (IRSS). Université Catholique de Louvain - Brussels, Belgium.

Guha-Sapir, D. V.; Below, F. R.; Ponserre, S. (2017). Annual Disaster Statistical Review 2016. Centre for Research on the Epidemiology of Disasters (CRED). Institute of Health and Society (IRSS). Université Catholique de Louvain - Brussels, Belgium.

Faria, F. L. F.; Guimaraes, G. V.; Bandeira, A. P. F.; Bandeira, R. A. (2014). Uma proposta de distribuição da cadeia de suprimentos na logística humanitária. Anais do XXVIII Congresso de Pesquisa e Ensino em Transportes, ANPET, Curitiba, v.1, 1-11.

Haddow, G. D.; Bullock, J. A.; Coppola, D. P. (2011). Introduction to emergency management. (3 (th $^{\text {th }}$.). Oxford, Butterworth-Heinemann.

Holguin-Veras, J.; Jaller, M.; Van Wassenhove, L.; Pérez, N.; Wachtendorf, T. (2012). On the unique features of postdisaster humanitarian logistics. Journal of Operations Management, v. 30 n. 7, p. 494-506. DOI: 10.1016/j.jom.2012.08.003

Jaller, M.; Holguín-Veras, J. (2011). Locating points of distribution in large urban disasters.. Transportation Research Board - 90th Annual Meeting, Washington, D.C. 
Kovács, G; Spens, K.M. (2012). Relief supply chain management for disasters: humanitarian aid and emergency logistics. IGI Global. Hershey PA, 2012. DOI: 10.1108/20426741211260769

Krawiec, B.; Kochersberger, K.; Conner, D. C. (2014). Autonomous aerial radio repeating using an based path planning approach. Journal of Intelligent \& Robotic Systems, v. 74, n. 3-4, p. 769-789. DOI: 10.1007/s10846-0139853-3

McCoy, J. H.; Lee, H. L. (2013). Using fairness models to improve equity in health delivery fleet management. Production and Operation Management Society, v. 23, n. 6, p. 965-977. DOI: 10.1111/poms.12101.

Meier, P. (2015). Digital humanitarians: how big data is changing the face of humanitarian response. (1 ${ }^{\text {st }}$ ed.). CRC Press.

North Carolina Emergency Managers Association (NCEMA). (2009). Commodity distribution and POD design. Deputy Log Chief - NC Division of Emergency Management.

United Nations Office for the Coordination of Humanitarian Affairs (OCHA). (2014). Policy Development and Studies Branch. Unmanned Aerial Vehicles in Humanitarian Response.

Overstreet, R. E.; Hall, D.; Hanna, J. B.; Rainer, R. K. (2011). Research in humanitarian logistics. Journal of Humanitarian Logistics and Supply Chain Management, v. 1, p.114-131. DOI: 10.1108/20426741111158421

Pan-American Health Organization (PAHO). (2000). Manual Logistical Management of Humanitarian Supply.

Silva, L. F. (2011). Gestão da logística humanitária: proposta de um referencial teórico. Dissertação de Mestrado. UFRJ/COPPEAD, Rio de Janeiro.

Souza, P. C.; Loureiro, C. F. (2014). Reflexões sobre os desastres ambientais no estado do rio de janeiro: questões socioambientais e psicossociais. Revista VITAS - Visões Transdisciplinares sobre Ambiente e Sociedade. ano IV, n. 8, p. 1-22. ISSN 2238-1627

Thomas, A.S. (2003). Humanitarian logistics: enabling disaster response. Fritz Institute.

Thomas, A. S., Kopczak, L. R. (2005). From logistics to supply chain management: The path forward in the humanitarian sector. Fritz Institute.

Tomasini, R.M; Van Wassenhove, L. N. (2004). A Framework to unravel, prioritize and coordinate vulnerability and complexity factors affecting a humanitarian response operation. Working Paper Series, INSEAD.

Van Wassenhove, LN. (2006). Blackett memorial lecture. Humanitarian aid logistics: supply chain management in high gear. Journal of the Operational Research Society, v. 57, p. 475-489. DOI: 10.1057/palgrave.jors.2602125

Yang, J.; Peng, C. (2014). Development of an UAS for post-earthquake disaster surveying and its application in Ms7.0 Lushan Earthquake, Sichuan, China. Computers \& Geosciences, v. 68, p. 22-30. DOI: j.cageo.2014.04.001 